\title{
MEDIUM-TERM EVOLUTION OF GHRELIN LEVELS ACCORDING TO THREE TYPES OF BARIATRIC SURGERIES AND ITS RELATION WITH HDL AND INSULIN RESISTANCE
}

\author{
${ }^{1}$ Concepción Santiago-Fernández, ${ }^{2,3}$ Sara García-Serrano, ${ }^{1}$ Mónica Tome, ${ }^{1,4}$ Francisco J. Tinahones, ${ }^{2,4}$ Eduardo García-Fuentes, ${ }^{1,4}$ Lourdes Garrido-Sánchez.
}

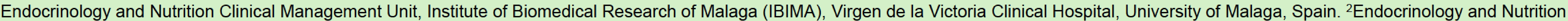
Clinical Management Unit, Institute of Biomedical Research of Malaga (IBIMA), Regional University Hospital, Malaga, Spain. ${ }^{3}$ CIBER of Diabetes and Metabolic Diseases (CIBERDEM), Malaga, Spain. ${ }^{4}$ CIBER of Physiopathology of Obesity and Nutrition (CIBEROBN), Malaga, Spain.

\section{Background}

Ghrelin is a gastrointestinal peptide involved in the regulation of body weight and energy balance. However, its behavior after bariatric surgery and its relationship with insulin resistance is still under discussion. The objective of this study is to perform a simultaneous evaluation of the association between changes in ghrelin levels and different variables after three types of bariatric surgery.

\section{Methods}

We analyzed ghrelin levels in 21 control non-obese and 103 morbidly obese subjects before and 6 months after bariatric surgery (Roux-en-Y gastric bypass (RYGB; $n=30$ ), biliopancreatic diversion of Scopinaro (BPD; $n=47)$ and sleeve gastrectomy (SG; $n=26)$ ).

\section{Results}

Before bariatric surgery ghrelin was decreased in morbidly obese subjects $(p<0.05)$. RYGB increased ghrelin $(p<0.05)$, BPD did not modify ghrelin and SG decreased ghrelin $(p<0.05)$ (Figure 1). The percent change in ghrelin levels $(\Delta$-ghrelin) was associated with the type of surgery in a multiple linear regression model $(p=0.017)$ (Figure 2). When we performed the same analysis only with those subjects in which the gastric fundus is maintained (RYGB and BPD), $\triangle$-ghrelin was negatively associated with $\Delta$-HOMA-IR ( $\mathrm{p}=0.001$ ) (Figure 3). The OR of having a lower $\Delta$-HOMA-IR of the persons with $\Delta$-ghrelin in Q1 quartile versus those with $\Delta$-ghrelin in Q4 quartile was $8.74(1.73-44.06)(p=0.009)$.

Table 1. Clinical and biochemical variables in non-obese and morbidly obese subjects before and 6 months after bariatric surgery.

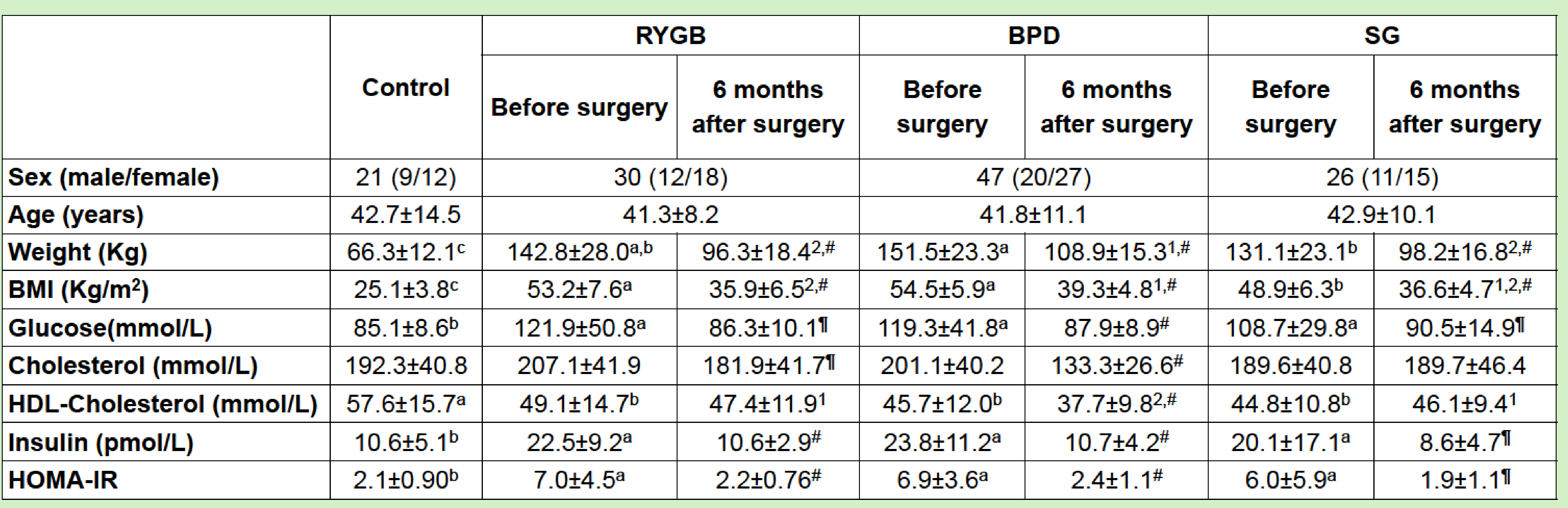

The results are given as the mean \pm SD. Different letters indicate significant differences between the means of the basal variables of morbidly obese subjects before surgery and non-obese subjects $(a, b$ and $c)(p<0.05)$. Different numbers indicate significant differences between the means of the three types of surgery in the variables analyzed 6 months after the bariatric surgery $(1$ and 2) $(p<0.05)$. Significant differences between before and after bariatric surgery into each type of bariatric surgery $\left({ }^{*} \mathrm{p}<0.05 ;\right.$; $\left.<0.01, \# p<0.001\right)$. BPD: Biliopancreatic diversion of Scopinaro, SG: Sleeve gastrectomy, RYGB: Rous-en-Y-gastric bypass, BMI: body mass index; HOMA-IR: homeostasis model assessment of insulin resistance index.

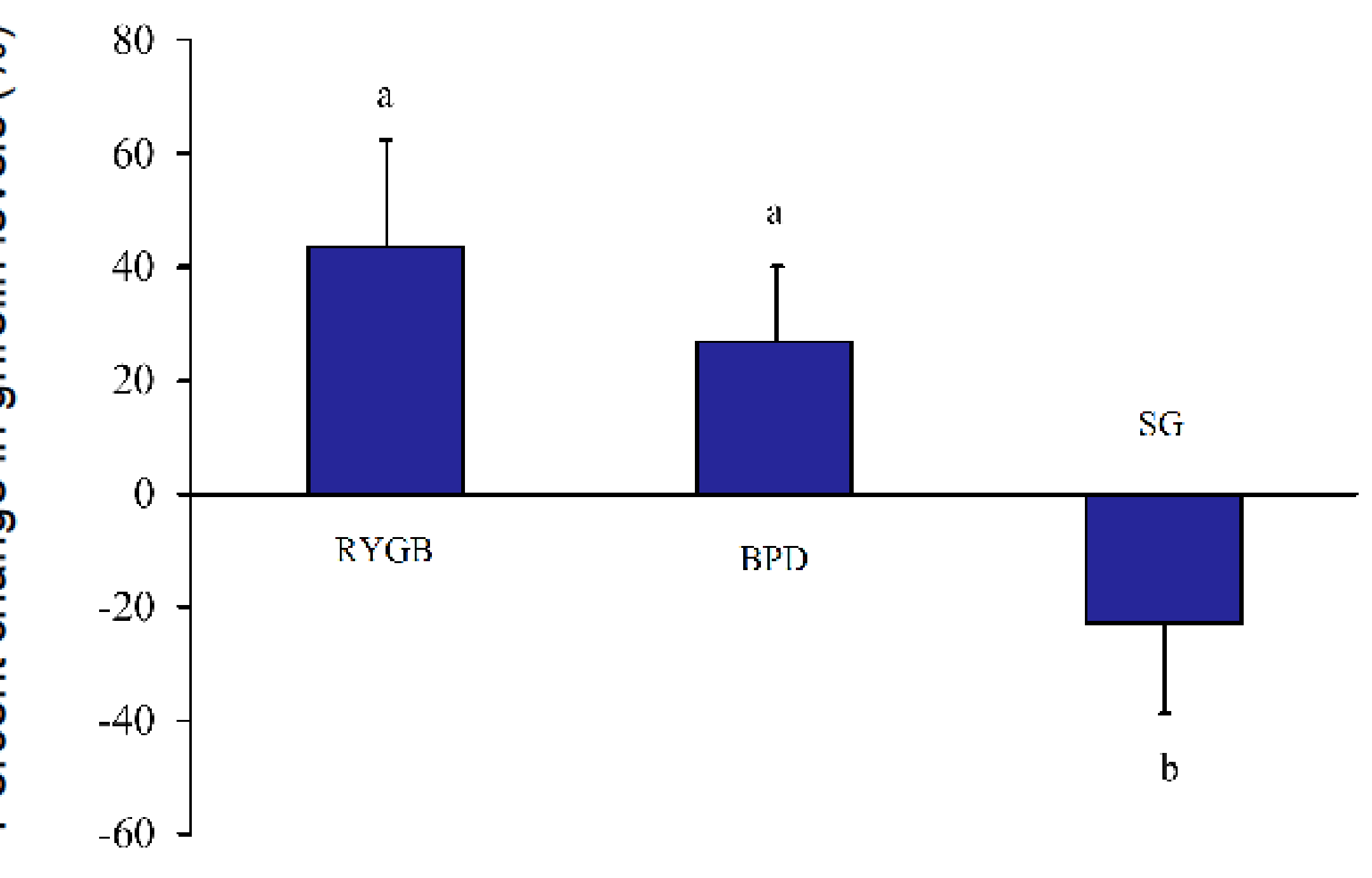

Figure 3. Percentage of change in serum ghrelin levels in morbidly obese subjects according to the type of bariatric surgery undergone (Roux-en-Y gastric bypass (RYGB), biliopancreatic diversion of Scopinaro (BPD) or sleeve gastrectomy (SG)) Data are presented as means \pm SEM. Different letters indicate significant differences between the means $(a$ and $b)(p<0.05)$.

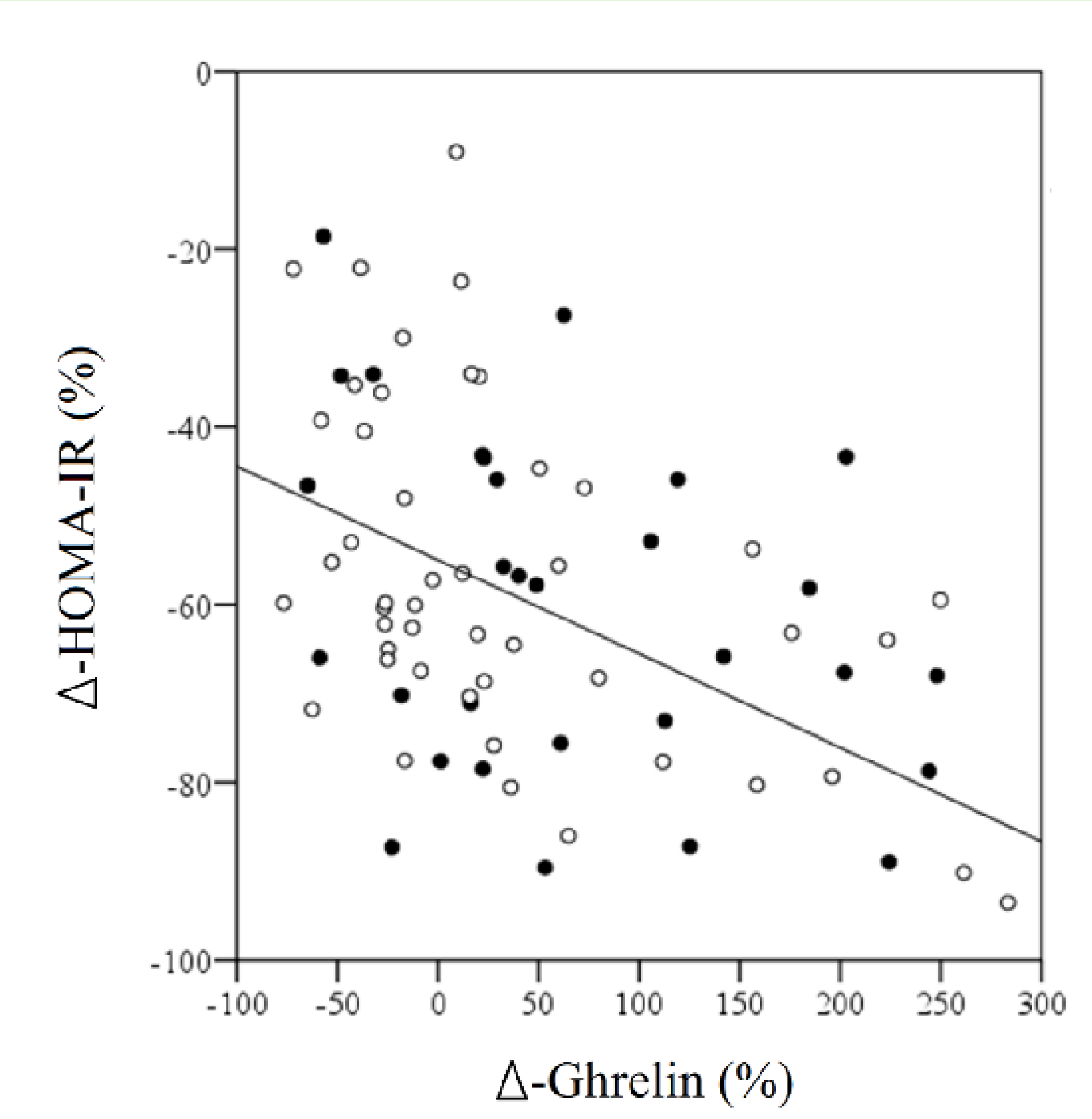

Figure 3. Association between the change in serum ghrelin levels $(\Delta$-ghrelina) and the change in HOMA-IR $(\Delta$-HOMA-IR) as a result of bariatric surgery (RYGB (๘) y BPD (口)).

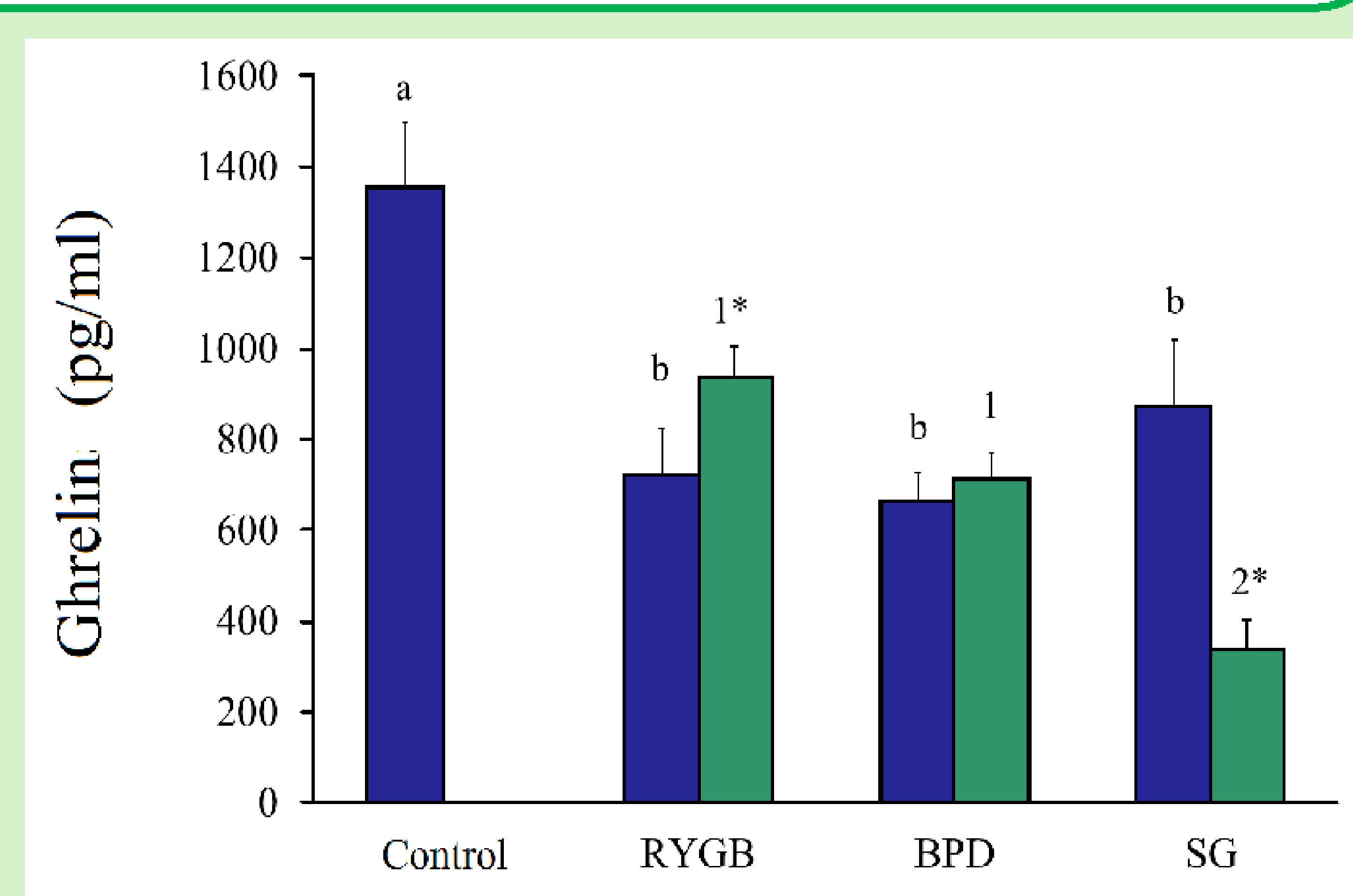

Figure 1. Serum ghrelin levels in non-obese and morbidly obese subjects before $(\square)$ and after bariatric surgery $(\square)$, according to the type of surgery undergone (Roux-en-Y gastric bypass (RYGB), biliopancreatic diversion of Scopinaro (BPD) or sleeve gastrectomy (SG)). Data are presented as means \pm SEM. Different letters indicate significant differences between the means of the basal ghrelin of morbidly obese subjects before surgery and nonobese subjects $(a$ and $b)(p<0.05)$. Different numbers indicate significant differences between the means of the three types of surgery in ghrelin levels 6 months after the bariatric surgery (1 and 2) $(p<0.05)$. Significant differences between before and after bariatric surgery into each type of bariatric surgery $\left({ }^{*} p<0.05\right)$

Table 2. Changes $(\Delta)$ in the anthropometric and biochemical variables 6 months after bariatric surgery according to the type of surgery undergone.

\begin{tabular}{|l|c|c|c|}
\hline & RYGB & BPD & SG \\
\hline$\Delta$-Weight & $-31.9 \pm 8.6^{\mathrm{a}}$ & $-27.7 \pm 6.8^{\mathrm{b}}$ & $-25.2 \pm 5.6^{\mathrm{b}}$ \\
\hline$\Delta$-BMI & $-31.9 \pm 8.6^{\mathrm{a}}$ & $-27.7 \pm 6.8^{\mathrm{b}}$ & $-25.2 \pm 5.6^{\mathrm{b}}$ \\
\hline $\boldsymbol{\Delta}$-Cholesterol & $-10.8 \pm 19.7^{\mathrm{b}}$ & $-32.5 \pm 13.1^{\mathrm{a}}$ & $0.9 \pm 22.7^{\mathrm{c}}$ \\
\hline$\Delta$-HDL-Cholesterol & $-1.01 \pm 25.1$ & $-13.1 \pm 38.2$ & $5.6 \pm 21.3$ \\
\hline$\Delta$-HOMA-IR & $-56.9 \pm 23.7$ & $-59.6 \pm 20.0$ & $-45.2 \pm 48.1$ \\
\hline
\end{tabular}

Data are presented as means \pm SD. BMI: body mass index; HOMA-IR: homeostasis model assessment of insulin resistance index. Differen letters indicate significant differences between the means $(p<0.05)$.

\section{Conclusions}

The changes in ghrelin levels after bariatric surgery are associated with the presence/absence of the gastric fundus. After bariatric surgery, the decrease of insulin resistance was associated with the increase of ghrelin levels in those techniques in which the fundus is not excluded.

\section{Acknowledgments}

This work was supported in part by a grant from the Instituto Salud Carlos III (PI12/00338) and the Consejería de Economía, Innovación, Ciencia y Empresa de la Junta de Andalucía. This study has been cofunded by FEDER funds. 